\title{
Stable and Accurate Marching-on-in-Time Solvers of Time Domain EFIE, MFIE, and CFIE based on Quasi-Exact Integration Technique
}

\author{
Xin Wang, Member, IEEE, Yifei Shi, Mingyu Lu, Senior Member, IEEE, Balasubramaniam Shanker, \\ Fellow, IEEE, Eric Michielssen, Fellow, IEEE, and Hakan Bağc1, Senior Member, IEEE
}

\begin{abstract}
The development of time domain integral equation solvers with robust late-time stability properties has been a long-standing quest. Among the many methods that have been investigated, those leveraging quasi-exact integration techniques appear to be the most successful. This paper presents stable and accurate marching-on-in-time (MOT) solvers for time domain electric, magnetic, and combined field integral equations (EFIE, MFIE, and CFIE) based on quasi-exact integration technique. The novel MOT solvers exhibit excellent stability while yielding highly accurate results, as demonstrated by various numerical examples. In addition, the solvers' excellent stability and accuracy properties are used to examine spurious modes encountered when time domain integral equations are applied to closed surfaces. It is demonstrated that MOT solutions to the time domain EFIE and MFIE often are polluted by spurious modes at the cavity's resonant frequencies whereas those of the CFIE solver are devoid of such contamination.
\end{abstract}

Index Terms-Electromagnetic transient scattering, time domain analysis, integral equations, moment methods, numerical stability

\section{INTRODUCTION}

$\mathrm{T}_{\mathrm{i}}$ ime domain electric, magnetic, and combined field integral equations (EFIE, MFIE, and CFIE) for analyzing

Manuscript received on April 9, 2020. This work was supported in part by National Natural Science Foundation of China Grant 61871220, National Natural Science Foundation of China Grant 61601207, and National Science Foundation Grant ECCS 1503600.

$\mathrm{X}$. Wang is with the College of Electronic and Information Engineering, Nanjing University of Aeronautics and Astronautics, Nanjing, Jiangsu 211106, China (e-mail: wang90@nuaa.edu.cn).

Y. Shi is with the Department of Electronic Engineering, Jiangsu University of Technology, Changzhou, Jiangsu 213000, China (e-mail: yifei.shi@jsut.edu.cn).

M. Lu is with the Department of Electrical and Computer Engineering, West Virginia University Institute of Technology, Beckley, WV 25801, USA (e-mail mingyu.lu@mail.wvu.edu).

B. Shanker is with the Department of Electrical and Computer Engineering, Michigan State University, East Lansing, MI 48824 USA (e-mail: bshanker@egr.msu.edu).

E. Michielssen is with the Department of Electrical Engineering and Computer Science, University of Michigan, Ann Arbor, MI 48109 USA (e-mail: emichiel@umich.edu).

H. Bağc1 is with the Division of Computer, Electrical, and Mathematical Sciences and Engineering, King Abdullah University of Science and Engineering, Thuwal 23955-6900, Saudi Arabia (e-mail: hakan.bagci@kaust.edu.sa). electromagnetic scattering from perfect electrically conducting (PEC) bodies have been studied since the 1970s [1-4]. Oftentimes, these equations are solved using marching-on-in-time (MOT) schemes [5]. Significant progress in the accuracy and efficiency of EFIE, MFIE, and CFIE MOT solvers notwithstanding [6], their late-time stability properties remain ill-understood [7], hampering the study of a range of other issues including the contamination of MOT solutions by spurious cavity modes [8].

The contributions of this paper are twofold, as elaborated one by one below.

First, it introduces novel and highly accurate time domain EFIE, MFIE, and CFIE solvers leveraging quasi-exact integration schemes. It also demonstrates these solvers' superior stability properties. Previous literature on the topic of stable time domain integral equation solvers alluded to the stability-enhancing impact of accurately evaluating MOT matrix elements [9-15]. Particularly, it was shown in [15] that when matrix elements arising in MOT-based EFIE solvers are evaluated "almost exactly" (that is, "exact for all practical purposes"), the resultant EFIE MOT solvers exhibit excellent late-time stability properties. This paper extends the scheme of [15] to the time domain MFIE and CFIE. As in [15], the unknown current density is discretized using Rao-Wilton-Glisson (RWG) spatial and shifted Lagrange temporal basis functions [16, 17], and time domain integral equations are discretized by Galerkin testing and point matching procedures in space and time. The proposed strategy for evaluating MOT matrix elements depends on the proximity of the basis and test triangles.

- When the basis and test triangles do not overlap, the (effectively) four-fold integrals in the expressions for EFIE, MFIE, and CFIE MOT matrix elements are cast in terms of three-dimensional integrals that are evaluated analytically and one-dimensional integral that is accurately and efficiently computed via numerical quadrature.

- When the basis and test triangles share one edge, the scheme for non-overlapping triangles is extended to account for the logarithmic singularity in the MFIE operator [18].

- When the basis and test triangles coincide, the MOT matrix elements are evaluated exactly, that is, without any approximation. While the analytic evaluation of MFIE matrix elements for this case is trivial, no exact scheme for 
evaluating EFIE matrix elements involving overlapping triangles has been reported before.

Numerical experiments demonstrate the late-time stability of EFIE, MFIE, and CFIE MOT solvers leveraging the proposed integration strategy. They also show that MOT solution accuracies can be tailored by adjusting the spatial and/or temporal discretization rates.

Second, this paper reports the outcome of an in-depth study into the susceptibility of solutions of EFIE, MFIE, and CFIE MOT solvers to spurious modes. It was conjectured in [4] that MOT solutions to the EFIE and MFIE may be contaminated by currents of modes supported by cavities formed by the scatterer surface; MOT solutions to the CFIE were presumed devoid of such spurious modes. This conjecture was supported by heuristic arguments for electromagnetics and acoustics [19, 20]. Theoretically, in continuous space-time, solutions to all TDIE formulations cannot be corrupted by spurious modes provided that the initial conditions stipulate zero fields before the simulation starts (at "time $=0$ " typically); however in the discrete world, it is conjectured that numerical errors may lead to the appearance of spurious modes. Unfortunately, the hypothesis in [4] has never been thoroughly corroborated by numerical experiments, in part because late-time instability issues prevented us from collecting sufficient late-time data in the past. This paper verifies the hypothesis in [4] by applying the proposed EFIE, MFIE, and CFIE MOT solvers (which are very stable and thus are able to generate sufficient late-time data) to several canonical geometries including a sphere, a circular cylinder, and a rectangular box. These canonical structures enable the explicit identification of spurious modes in MOT solutions of the EFIE and MFIE, as well as the absence thereof in those of the CFIE. Therefore, the numerical results in this paper verify the hypothesis in [4].

This paper is organized as follows. Section II describes the novel EFIE, MFIE, and CFIE MOT solvers leveraging quasi-exact schemes to evaluate MOT matrix elements. Section III presents numerical results that demonstrate the EFIE, MFIE, and CFIE MOT solvers' accuracy and late-time stability; in addition, it presents a comprehensive analysis of susceptibility of the MOT solutions to spurious modes. Section IV summarizes our findings.

\section{MOT SOLVERS BASED ON QUASI-EXACT INTEGRATION \\ TECHNIQUE FOR TIME DOMAIN EFIE, MFIE, AND CFIE}

Consider a PEC scatterer with closed surface $S$ that resides in free space (Fig. 1), and is illuminated by electric and magnetic fields $\mathbf{E}^{i}$ and $\mathbf{H}^{i}$. The current density $\mathbf{J}$ induced on $S$ generates scattered electric and magnetic fields $\mathbf{E}^{s}$ and $\mathbf{H}^{S}$ that satisfy the CFIE

$$
\begin{aligned}
& -\xi \hat{\mathbf{n}}\left(\mathbf{r}_{o}\right) \times \hat{\mathbf{n}}\left(\mathbf{r}_{o}\right) \times\left\{\mathbf{E}^{i}\left(\mathbf{r}_{o}, t\right)+\mathbf{E}^{S}\left(\mathbf{r}_{o}, t\right)\right\} \\
& +(1-\xi) \eta_{0} \hat{\mathbf{n}}\left(\mathbf{r}_{o}\right) \times\left\{\mathbf{H}^{i}\left(\mathbf{r}_{o}, t\right)+\mathbf{H}^{S}\left(\mathbf{r}_{o}, t\right)\right\}=\mathbf{0}
\end{aligned}, \mathbf{r}_{o} \in S_{-},
$$

where

$$
\begin{aligned}
\mathbf{E}^{S}\left(\mathbf{r}_{o}, t\right) & =\frac{-\mu_{0}}{4 \pi} \iint_{S} d s \frac{\left[\frac{\partial}{\partial t^{\prime}} \mathbf{J}\left(\mathbf{r}_{S}, t^{\prime}\right)\right]_{t^{\prime}=t-R / c}}{R} \\
& +\frac{1}{4 \pi \varepsilon_{0}} \nabla_{o} \iint_{S} d s \frac{\int_{-\infty}^{t-R / c} d t^{\prime}\left[\nabla_{s} \cdot \mathbf{J}\left(\mathbf{r}_{S}, t^{\prime}\right)\right]}{R}
\end{aligned}
$$

and

$$
\mathbf{H}^{S}\left(\mathbf{r}_{o}, t\right)=\frac{1}{4 \pi} \nabla_{o} \times \iint_{S} d s \frac{\left[\mathbf{J}\left(\mathbf{r}_{s}, t^{\prime}\right)\right]_{t^{\prime}=t-R / c}}{R} .
$$

In the above equations, $\varepsilon_{0}$ and $\mu_{0}$ are the free-space permittivity and permeability, $\eta_{0}=\sqrt{\mu_{0} / \varepsilon_{0}}$ and $c=1 / \sqrt{\varepsilon_{0} \mu_{0}}$ are the free-space intrinsic impedance and speed of light, and $0 \leq \xi \leq 1$. Additionally, $\mathbf{r}_{o}$ denotes an observation point on $S_{-}$, a surface residing just inside $S$ (Fig. $1), \mathbf{r}_{S}$ denotes a source point on $S, \nabla_{o}$ and $\nabla_{S}$ act on $\mathbf{r}_{o}$ and $\mathbf{r}_{S}, R=\left|\mathbf{r}_{o}-\mathbf{r}_{S}\right|$, and $\hat{\mathbf{n}}$ is the outward normal direction to $S$. The EFIE and MFIE are special cases of CFIE with $\xi=1$ and $\xi=0$, respectively. The incident fields and current density are assumed vanishingly small on $S$ for $t<0$.



Fig. 1. Scattering problem under consideration.

To solve (1), $S$ is approximated by flat triangles and $\mathbf{J}$ is expanded in terms of $N_{s}$ spatial and $N_{t}$ temporal basis functions as

$$
\mathbf{J}\left(\mathbf{r}_{S}, t\right)=\sum_{n=1}^{N_{S}} \sum_{l=1}^{N_{t}} J_{n}^{l} \boldsymbol{\Lambda}_{n}\left(\mathbf{r}_{S}\right) T_{l}(t) .
$$

Here, $\boldsymbol{\Lambda}_{n}\left(\mathbf{r}_{s}\right)\left(n=1,2, \ldots, N_{s}\right)$ is the RWG spatial basis function associated with the triangular mesh's $n$-th edge, and $T_{l}(t)=T(t-l \Delta t)\left(l=1,2, \ldots, N_{t}\right.$, and $\Delta t$ is the time step size $)$ is a shifted Lagrange temporal interpolant [15-17]. Substituting (4) into (1), testing the resulting equation in space using functions $\Lambda_{m}\left(\mathbf{r}_{o}\right)\left(m=1,2, \ldots, N_{s}\right)$, and testing the equation in time at $t=t_{k}=k \Delta t$ yield the following MOT equation:

$$
\overline{\mathbf{Z}}^{0} \mathbf{J}^{k}=\mathbf{V}^{k}-\sum_{l=1}^{k-1} \overline{\mathbf{Z}}^{k-l} \mathbf{J}^{l} .
$$

In (5), $\mathbf{J}^{k}=\left[J_{1}^{k}, J_{2}^{k}, \cdots, J_{N_{s}}^{k}\right]^{T}\left(k=1,2, \ldots, N_{t}\right), \mathbf{V}^{k}$ is an $N_{S}$-vector with elements 


$$
\begin{aligned}
{\left[\mathbf{V}^{k}\right]_{m} } & =-\xi \iint_{S_{-}} \Lambda_{m}\left(\mathbf{r}_{o}\right) \cdot \mathbf{E}^{i}\left(\mathbf{r}_{o}, t_{k}\right) d s \\
& -(1-\xi) \eta_{0} \iint_{S_{-}} \Lambda_{m}\left(\mathbf{r}_{o}\right) \cdot \hat{\mathbf{n}}\left(\mathbf{r}_{o}\right) \times \mathbf{H}^{i}\left(\mathbf{r}_{o}, t_{k}\right) d s
\end{aligned}
$$

and $\overline{\mathbf{Z}}^{l}, l=0,1,2, \ldots, N_{t}-1$, are $N_{s} \times N_{s}$ matrices with elements

$$
\begin{aligned}
& {\left[\overline{\mathbf{Z}}^{l}\right]_{m n}=}-\xi \frac{\mu_{0}}{4 \pi} \iint_{S_{-}} d s \Lambda_{m}\left(\mathbf{r}_{o}\right) \cdot \\
& \iint_{S} d s \Lambda_{n}\left(\mathbf{r}_{S}\right) \frac{\left[\frac{d}{d t^{\prime}} T\left(t^{\prime}\right)\right]_{t^{\prime}=t_{l}-R / c}}{R} \\
&-\xi \frac{1}{4 \pi \varepsilon_{0}} \iint_{S_{-}} d s\left[\nabla_{o} \cdot \Lambda_{m}\left(\mathbf{r}_{o}\right)\right] \\
& \iint_{S} d s\left[\nabla_{S} \cdot \Lambda_{n}\left(\mathbf{r}_{S}\right)\right] \frac{\int_{-\infty}^{t_{l}-R / c} T\left(t^{\prime}\right) d t^{\prime}}{R} \\
&+(1-\xi) \frac{\eta_{0}}{4 \pi} \iint_{S} d s \Lambda_{m}\left(\mathbf{r}_{o}\right) \cdot \hat{\mathbf{n}}\left(\mathbf{r}_{o}\right) \times \nabla_{o} \times \\
& \iint_{S} d s \Lambda_{n}\left(\mathbf{r}_{s}\right) \frac{T\left(t_{l}-R / c\right)}{R}
\end{aligned}
$$

The MOT equations in (5) can be solved for $\mathbf{J}^{k}$ once the current coefficient vectors $\mathbf{J}^{l}, l<k$ are known. In what follows, a procedure to accurately compute the matrix elements in (7) is outlined.

An RWG basis function $\Lambda_{m}\left(\mathbf{r}_{o}\right)\left(\Lambda_{n}\left(\mathbf{r}_{s}\right)\right)$ spans two triangles adjacent to edge $m(n)$ [16]. On each of these triangles, $\Lambda_{m}\left(\mathbf{r}_{o}\right)\left(\Lambda_{n}\left(\mathbf{r}_{s}\right)\right)$ is proportional to $\mathbf{r}_{o}-\mathbf{r}_{o b}\left(\mathbf{r}_{s}-\mathbf{r}_{s b}\right)$, where $\mathbf{r}_{o b}\left(\mathbf{r}_{s b}\right)$ represents the triangle's free vertex. Moreover, on each of the triangles, $\nabla_{o} \cdot \Lambda_{m}\left(\mathbf{r}_{o}\right)\left(\nabla_{s} \cdot \Lambda_{n}\left(\mathbf{r}_{s}\right)\right)$ is constant. It follows that the matrix elements in (7) can be expressed as linear combinations of the following three generic integrals.

$$
\begin{gathered}
I_{1}^{E F I E}=\iint_{P_{o}} d s\left(\mathbf{r}_{o}-\mathbf{r}_{o b}\right) \cdot \\
\iint_{P_{S}} d s\left(\mathbf{r}_{s}-\mathbf{r}_{s b}\right) \frac{\left[\frac{d}{d t^{\prime}} T\left(t^{\prime}\right)\right]_{t^{\prime}=t_{l}-R / c}}{R}, \\
I_{2}^{E F I E}=\iint_{P_{o}} d s \iint_{P_{S}} d s \frac{\int_{-\infty}^{t_{-}-R / c} T\left(t^{\prime}\right) d t^{\prime}}{R}, \\
I^{M F I E}=\iint_{P_{o}} d s\left(\mathbf{r}_{o}-\mathbf{r}_{o b}\right) \cdot \hat{\mathbf{n}}_{o} \times \nabla_{o} \times \\
\quad \iint_{P_{S}} d s\left(\mathbf{r}_{s}-\mathbf{r}_{s b}\right) \frac{T\left(t_{l}-R / c\right)}{R} .
\end{gathered}
$$

Integrals of type (8) and type (9) contribute to the EFIE portion of $\left[\overline{\mathbf{Z}}^{l}\right]_{m n}$ (the first two terms on the right-hand-side of (7)) while integrals of type (10) add to the MFIE portion of
$\left[\overline{\mathbf{Z}}^{l}\right]_{m n}$ (the third term on the right-hand-side of (7)). In (8) to (10), $P_{o}$ and $P_{S}$ denote triangular patches containing the observation and source points $\mathbf{r}_{o}$ and $\mathbf{r}_{s} ; \mathbf{r}_{o b}$ and $\mathbf{r}_{s b}$ represent a vertex of $P_{o}$ and $P_{S}$; and $\hat{\mathbf{n}}_{o}$ is the direction normal to $P_{o}$.

To evaluate integrals (8) to (10) accurately, three scenarios are considered:

A. Disjoint triangles: $P_{o}$ and $P_{S}$ do not share any edge but may share one node.

B. Neighboring triangles: $P_{O}$ and $P_{S}$ share one edge.

C. Overlapping triangles: $P_{o}$ and $P_{S}$ coincide.

\section{A. Disjoint triangles}

To evaluate (8) to (10) for disjoint triangles $P_{o}$ and $P_{S}$, consider the Cartesian coordinate system $(u, v, w)$ introduced in Fig. 2, in which the $w$ axis is perpendicular to $P_{S}$ and the $u$ axis is parallel to $\hat{\mathbf{w}} \times \hat{\mathbf{n}}_{o}$.

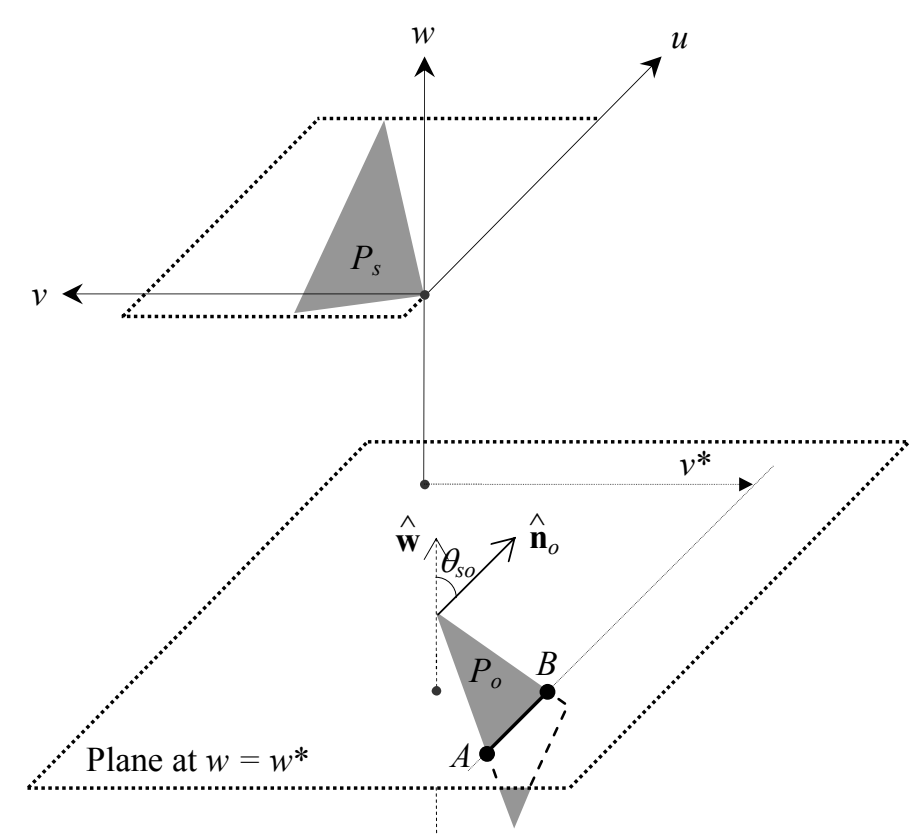

Fig. 2. Illustration of quasi-exact integration technique in the "disjoint triangles" case.

In the $(u, v, w)$ coordinate system, any two-dimensional integration over $P_{o}$ can be expressed as

$$
\iint_{P_{O}} d s\{\cdots\}=\frac{1}{\sin \left(\theta_{s o}\right)} \int d w^{*} \int_{\mathrm{AB}} d u_{o}\{\cdots\},
$$

where $\{\cdots\}$ represents the integrand and $\overline{\mathrm{AB}}$ is a line segment on $P_{o}$ parallel to the $u$-axis at $w=w^{*}$ (Fig. 2). Here, $\theta_{s o}$ is the angle between $\hat{\mathbf{n}}_{o}$ and $\hat{\mathbf{w}}$. The $v$ coordinate of line segment $\overline{\mathrm{AB}}$ is a constant and denoted as $v^{*}$.

Following (11), the integrals in (8) to (10) are re-arranged as

$$
\begin{aligned}
& I_{1}^{E F I E}=\frac{1}{\sin \left(\theta_{\text {so }}\right)} \int d w^{*} \Phi_{1}^{E F I E}\left(w^{*}\right), \\
& I_{2}^{E F I E}=\frac{1}{\sin \left(\theta_{\text {so }}\right)} \int d w^{*} \Phi_{2}^{E F I E}\left(w^{*}\right),
\end{aligned}
$$




$$
I^{M F I E}=\frac{1}{\sin \left(\theta_{s o}\right)} \int d w^{*} \Phi^{M F I E}\left(w^{*}\right)
$$

where

$$
\begin{aligned}
& \Phi_{1}^{E F I E}\left(w^{*}\right)=\int_{\mathrm{AB}} d u_{o}\left(\mathbf{r}_{o}-\mathbf{r}_{o b}\right) \cdot \\
& \iint_{P_{S}} d s\left(\mathbf{r}_{s}-\mathbf{r}_{s b}\right) \frac{\left[\frac{d}{d t^{\prime}} T\left(t^{\prime}\right)\right]_{t^{\prime}=t_{l}-R / c}}{R}, \\
& \Phi_{2}^{E F I E}\left(w^{*}\right)=\int_{\mathrm{AB}} d u_{o} \iint_{P_{S}} d s \frac{\int_{-\infty}^{t_{l}-R / c} T\left(t^{\prime}\right) d t^{\prime}}{R},
\end{aligned}
$$

and

$$
\begin{aligned}
\Phi^{M F I E}\left(w^{*}\right)=\int_{\mathrm{AB}} d u_{o}\left(\mathbf{r}_{o}-\mathbf{r}_{o b}\right) \cdot \hat{\mathbf{n}}_{o} \times \nabla_{o} \times \\
\quad \iint_{P_{S}} d s\left(\mathbf{r}_{s}-\mathbf{r}_{s b}\right) \frac{T\left(t_{l}-R / c\right)}{R} .
\end{aligned}
$$

The integrals $\Phi_{1}^{E F I E}, \Phi_{2}^{E F I E}$, and $\Phi^{M F I E}$ in (15)-(17) can be evaluated analytically. To this end, two indicator functions are defined as

$$
\begin{aligned}
& \Pi^{1 \mathrm{D}}\left(u_{o}\right)= \begin{cases}1 & u_{o} \in \overline{\mathrm{AB}} \\
0 & u_{o} \notin \overline{\mathrm{AB}}\end{cases} \\
& \Pi^{2 \mathrm{D}}\left(u_{s}, v_{s}\right)=\left\{\begin{array}{ll}
1 & \left(u_{s}, v_{s}\right) \in P_{s} \\
0 & \left(u_{s}, v_{s}\right) \notin P_{s}
\end{array} .\right.
\end{aligned}
$$

With the aid of the two indicator functions, the integrals in (15)-(17) can be expressed as

$$
\begin{aligned}
\Phi_{1}^{E F I E}\left(w^{*}\right)= & \int_{-\infty}^{+\infty} \int_{-\infty}^{+\infty} \int_{-\infty}^{+\infty} d u_{o} d v_{o} d w_{o} \delta\left(w_{o}-w^{*}\right) \delta\left(v_{o}-v^{*}\right) \Pi^{1 \mathrm{D}}\left(u_{o}\right) \\
& \int_{-\infty}^{+\infty} \int_{-\infty}^{+\infty} \int_{-\infty}^{+\infty} d u_{s} d v_{s} d w_{s} \\
\Phi_{2}^{E F I E}\left(w^{*}\right)= & \left.\int_{-\infty}^{+\infty} \int_{-\infty}^{+\infty} \int_{-\infty}^{+\infty} d u_{o} d v_{o} d w_{o}, v_{s}\right) \\
& \int_{-\infty}^{+\infty} \int_{-\infty}^{+\infty} \delta\left(w_{o}-w^{*}\right) \delta\left(v_{o}-v^{*}\right) \Pi^{1 \mathrm{D}}\left(u_{o}\right) \\
& \left.\mathbf{r}_{o}\right) \cdot\left(\mathbf{r}_{s}-\mathbf{r}_{s b}\right) \frac{\left.\left[t^{\prime}\right)\right]}{R} d v_{t^{\prime}=t_{l}-R / c}
\end{aligned}
$$

and

$$
\begin{aligned}
\Phi^{M F I E}\left(w^{*}\right)= & \int_{-\infty}^{+\infty} \int_{-\infty}^{+\infty} \int \frac{\infty}{-\infty} d u_{o} d v_{o} d w_{o} \\
& \left.\int_{-\infty}^{+\infty} \int_{-\infty}^{+\infty} \int_{-\infty}^{+\infty} d w_{o}-w^{*}\right) \delta\left(v_{o}-v^{*}\right) \Pi^{1 \mathrm{D}}\left(u_{o}\right) \\
& \delta\left(w_{s}\right) \Pi^{2 \mathrm{D}}\left(u_{s}, v_{s}\right) \\
& \left\{-\frac{T\left(t_{l}-R / c\right)}{R^{3}}-\frac{1}{c R^{2}}\left[\frac{d T\left(t^{\prime}\right)}{\partial t^{\prime}}\right]_{t^{\prime}=t_{l}-R / c} \times\left(\mathbf{r}_{o}-\mathbf{r}_{s}\right) \times\left(\mathbf{r}_{s}-\mathbf{r}_{s b}\right)\right.
\end{aligned}
$$

In (20) to (22), $\quad \mathbf{r}_{o}=u_{o} \hat{\mathbf{u}}+v_{o} \hat{\mathbf{v}}+w_{o} \hat{\mathbf{w}} \quad$ and $\mathbf{r}_{s}=u_{s} \hat{\mathbf{u}}+v_{s} \hat{\mathbf{v}}+w_{s} \hat{\mathbf{w}}$ are expressed in the $(w, v, u)$ coordinate system. Using variable transformations $u=u_{o}-u_{s}$, $v=v_{o}-v_{s}$, and $w=w_{o}-w_{s}$, the integrals in (20) to (22) become

$$
\begin{gathered}
\Phi_{1}^{E F I E}\left(w^{*}\right)=\int_{-\infty}^{+\infty} \int_{-\infty}^{+\infty} \int_{-\infty}^{+\infty} d u d v d w \Omega_{1}^{E F I E}(u, v, w) \\
\frac{\left[\frac{d}{d t^{\prime}} T\left(t^{\prime}\right)\right]_{t^{\prime}=t_{l}-r / c}}{r}, \\
\Phi_{2}^{E F I E}\left(w^{*}\right)=\int_{-\infty}^{+\infty} \int_{-\infty}^{+\infty} \int_{-\infty}^{+\infty} d u d v d w \Omega_{2}^{E F I E}(u, v, w), \\
\int_{-\infty}^{t_{l}-r / c} \frac{T\left(t^{\prime}\right)}{r} d t^{\prime}
\end{gathered}
$$

and

$$
\begin{aligned}
\Phi^{M F I E}\left(w^{*}\right)= & \int_{-\infty}^{+\infty} \int_{-\infty}^{+\infty} \int_{-\infty}^{+\infty} d u d v d w \Omega^{M F I E}(u, v, w) \\
& \left\{-\frac{T\left(t_{l}-r / c\right)}{r^{3}}-\frac{1}{c r^{2}}\left[\frac{d T\left(t^{\prime}\right)}{\partial t^{\prime}}\right]_{t^{\prime}=t_{l}-r / c}\right\},
\end{aligned}
$$

where

$$
\begin{aligned}
& \Omega_{1}^{E F I E}(u, v, w)=\int_{-\infty}^{+\infty} \int_{-\infty}^{+\infty} \int_{-\infty}^{+\infty} d u_{o} d v_{o} d w_{o} \\
& \delta\left(w_{o}-w^{*}\right) \delta\left(v_{o}-v^{*}\right) \Pi^{1 \mathrm{D}}\left(u_{o}\right), \\
& \delta\left(w_{o}-w\right) \Pi^{2 \mathrm{D}}\left(u_{o}-u, v_{o}-v\right) \\
& \left(\mathbf{r}_{o}-\mathbf{r}_{o b}\right) \cdot\left(\mathbf{r}_{o}-\mathbf{r}-\mathbf{r}_{s b}\right) \\
& \Omega_{2}^{E F I E}(u, v, w)=\int_{-\infty}^{+\infty} \int_{-\infty}^{+\infty} \int_{-\infty}^{+\infty} d u_{o} d v_{o} d w_{o} \\
& \delta\left(w_{o}-w^{*}\right) \delta\left(v_{o}-v^{*}\right) \Pi^{1 \mathrm{D}}\left(u_{o}\right), \\
& \delta\left(w_{o}-w\right) \Pi^{2 \mathrm{D}}\left(u_{o}-u, v_{o}-v\right)
\end{aligned}
$$




$$
\begin{aligned}
\Omega^{M F I E}(u, v, w)=\int_{-\infty}^{+\infty} \int_{-\infty}^{+\infty} \int_{-\infty}^{+\infty} d u_{o} d v_{o} d w_{o} \\
\quad \delta\left(w_{o}-w^{*}\right) \delta\left(v_{o}-v^{*}\right) \Pi^{1 \mathrm{D}}\left(u_{o}\right) \\
\quad \delta\left(w_{o}-w\right) \Pi^{2 \mathrm{D}}\left(u_{o}-u, v_{o}-v\right) \\
\left(\mathbf{r}_{o}-\mathbf{r}_{o b}\right) \cdot \hat{\mathbf{n}}_{o} \times \mathbf{r} \times\left(\mathbf{r}_{o}-\mathbf{r}-\mathbf{r}_{s b}\right)
\end{aligned}
$$

$\mathbf{r}=u \hat{\mathbf{u}}+v \hat{\mathbf{v}}+w \hat{\mathbf{w}}$, and $r=\sqrt{u^{2}+v^{2}+w^{2}}$.

Three important observations pertinent to (23)-(28) are presented next; their rigorous proofs are straightforward and hence not detailed here.

First, $\Omega_{1}^{E F I E}(u, v, w), \Omega_{2}^{E F I E}(u, v, w)$, and $\Omega^{M F I E}(u, v, w)$ represent spatial correlations between $\overline{\mathrm{AB}}$ and $P_{S}$. Out of the three-fold integrations in (26), (27), and (28), the two-fold integrations with respect to $v_{o}$ and $w_{o}$ are easily evaluated in closed form due to the presence of Dirac delta functions in the integrands. The analytical evaluation of the remaining one-dimensional integral with respect to $u_{o}$ can be performed analytically as well. Thus, starting from (26)-(28) analytical expressions for $\Omega_{1}^{E F I E}(u, v, w), \Omega_{2}^{E F I E}(u, v, w)$, and $\Omega^{M F I E}(u, v, w)$ can be obtained. All three quantities equate to zero when $w \neq w^{*}$ and when $(u, v)$ is outside a polygonal region. Therefore, the integrals in (23), (24), and (25) all can be interpreted as fields at the spatial origin produced by a polygonal source located in the plane $w=w^{*}$.

Second, when $\Omega_{1}^{E F I E}(u, v, w), \Omega_{2}^{E F I E}(u, v, w)$, and $\Omega^{M F I E}(u, v, w)$ are non-zero, they can be expressed as multinomials involving terms of the form $u^{i} v^{j}$, where $i$ and $j$ are integers with values of $0,1,2, \ldots$ This fact is quite obvious by examining the integrands of (26), (27), and (28).

Third, $T(t)$ is a piecewise-polynomial function. As a result, $T\left(t_{l}-r / c\right),\left[d T\left(t^{\prime}\right) / d t^{\prime}\right]_{t^{\prime}=t_{l}-r / c}$, and $\int_{-\infty}^{t_{l}-r / c} T\left(t^{\prime}\right) d t^{\prime}$ can be expressed as linear combinations of $r^{\tau}$, where $\tau$ is an integer that takes on values $0,1,2, \ldots$

In view of the above three observations, $\Phi_{1}^{E F I E}, \Phi_{2}^{E F I E}$, and $\Phi^{M F I E}$ in (23) to (25) can be decomposed into linear combinations of integrals of the form

$\iint\left(r^{\tau} u^{i} v^{j}\right) d u d v, \tau=-3,-1,0,1,2, \ldots$ and $i, j=0,1,2, \ldots$

All the integrations in (29) can be evaluated analytically by following the procedure in [15]. Consequently, $\Phi_{1}^{E F I E}$, $\Phi_{2}^{E F I E}$, and $\Phi^{M F I E}$ all can be evaluated in closed form.

After $\Phi_{1}^{E F I E}, \Phi_{2}^{E F I E}$, and $\Phi^{M F I E}$ are evaluated analytically, the one-dimensional integration along $w^{*}$ in (12) to (14) is evaluated numerically using standard Gauss-Legendre integration rule with $N_{w}$ quadrature points. When the edge length of triangles does not exceed one-tenth of the free-space wavelength corresponding to the highest frequency appreciably present in the incident fields, a 7-point Gauss-Legendre rule $\left(N_{w}=7\right)$ typically yields at least 3 digits of precision.

\section{B. Neighboring triangles}

The procedure in Subsection A for evaluating integrals
(8)-(10) for disjoint triangles by and large carries over to the case of "neighboring triangles", with the exception of a singular term in the MFIE operator that must be extracted to achieve the optimal accuracy.

Using the coordinate system $(u, v, w)$ in Fig. 3, integrals $I_{1}^{E F I E}, I_{2}^{E F I E}$, and $I^{M F I E}$ once again are rearranged as in (12)-(14) and the integrands $\Phi_{1}^{E F I E}, \Phi_{2}^{E F I E}$, and $\Phi^{M F I E}$ in (12)-(14) are evaluated analytically as described in Subsection A. The integrations along $w^{*}$ in (12) and (13) can be evaluated accurately by standard numerical integration rules. However, direct numerical integration along $w^{*}$ in (14) yields poor accuracy because of the presence of a logarithmic singularity in $\Phi^{M F I E}$.



Fig. 3. Illustration of quasi-exact integration technique in the "neighboring triangles" case.

By defining $\breve{\mathbf{H}}$ as

$$
\breve{\mathbf{H}}\left(\mathbf{r}_{o}\right)=\nabla_{o} \times \iint_{P_{S}} d s\left(\mathbf{r}_{s}-\mathbf{r}_{s b}\right) \frac{T\left(t_{l}-R / c\right)}{R},
$$

$\Phi^{M F I E}$ in (17) can be expressed as

$$
\Phi^{M F I E}\left(w^{*}\right)=\int_{\mathrm{AB}} d u_{o}\left(\mathbf{r}_{o}-\mathbf{r}_{o b}\right) \cdot \hat{\mathbf{n}}_{o} \times \breve{\mathbf{H}}\left(\mathbf{r}_{o}\right) .
$$

Here, $\breve{\mathbf{H}}$ is the magnetic field produced by a surface electric current residing on $P_{S}$ and observed at $\mathbf{r}_{o}=u_{o} \hat{\mathbf{u}}+v^{*} \hat{\mathbf{v}}+w^{*} \hat{\mathbf{w}}$. Its singular behavior when $l=0$ and $w^{*} \rightarrow 0$ has been studied extensively in the literature (see, for example, [18]):

$$
\breve{\mathbf{H}}\left(\mathbf{r}_{o}\right) \stackrel{w^{*} \rightarrow 0}{\longrightarrow}-2\left(\mathbf{r}_{o}-\mathbf{r}_{s b}\right) \times \hat{\mathbf{v}} \ln \left(w^{*}\right) .
$$

Consequently,

$$
\begin{aligned}
\Phi^{M F I E}\left(w^{*}\right) \stackrel{w^{*} \rightarrow 0}{\longrightarrow} \frac{\int}{\mathrm{A}_{0} \mathrm{~B}_{0}} d u_{o}\left(\mathbf{r}_{o}-\mathbf{r}_{o b}\right) \cdot \hat{\mathbf{n}}_{o} \times \\
\quad\left[-2\left(\mathbf{r}_{o}-\mathbf{r}_{s b}\right) \times \hat{\mathbf{v}} \ln \left(w^{*}\right)\right], \\
=\kappa \ln \left(w^{*}\right)
\end{aligned}
$$

where $\overline{\mathrm{A}_{0} \mathrm{~B}_{0}}$ is the edge shared by $P_{o}$ and $P_{s}$, and 


$$
\begin{aligned}
\kappa & =-2 \frac{\int}{\mathrm{A}_{0} \mathrm{~B}_{0}} d u_{o}\left(\mathbf{r}_{o}-\mathbf{r}_{o b}\right) \cdot \hat{\mathbf{n}}_{o} \times\left(\mathbf{r}_{o}-\mathbf{r}_{s b}\right) \times \hat{\mathbf{v}} \\
& =-2\left(\hat{\mathbf{n}}_{o} \cdot \hat{\mathbf{v}}\right) \frac{\int}{\mathrm{A}_{0} \mathrm{~B}_{0}} d u_{o}\left(\mathbf{r}_{o}-\mathbf{r}_{o b}\right) \cdot\left(\mathbf{r}_{o}-\mathbf{r}_{s b}\right) . \\
& +2 \frac{\int}{\mathrm{A}_{0} \mathrm{~B}_{0}} d u_{o}\left\{\left(\mathbf{r}_{o}-\mathbf{r}_{o b}\right) \cdot \hat{\mathbf{v}}\right\}\left\{\hat{\mathbf{n}}_{o} \cdot\left(\mathbf{r}_{o}-\mathbf{r}_{s b}\right)\right\}
\end{aligned}
$$

The derivation in (34) relies on the vector identity $\hat{\mathbf{n}}_{o} \times\left(\mathbf{r}_{o}-\mathbf{r}_{s b}\right) \times \hat{\mathbf{v}}=\left(\mathbf{r}_{o}-\mathbf{r}_{s b}\right)\left(\hat{\mathbf{n}}_{o} \cdot \hat{\mathbf{v}}\right)-\hat{\mathbf{v}}\left\{\hat{\mathbf{n}}_{o} \cdot\left(\mathbf{r}_{o}-\mathbf{r}_{s b}\right)\right\}$. The analytical evaluation of $\kappa$ is straightforward because $\left(\hat{\mathbf{r}}_{o} \cdot \hat{\mathbf{n}}_{o}\right)$ and $\left(\mathbf{r}_{o} \cdot \hat{\mathbf{v}}\right)$ are constants along $\widehat{\mathrm{A}_{0} \mathrm{~B}_{0}}$. Having identified the logarithmic singularity in (33), integral $I^{M F I E}$ in (14) is written as

$$
\begin{aligned}
I^{M F I E} & =\frac{1}{\sin \left(\theta_{\text {so }}\right)} \int d w^{*}\left\{\Phi^{M F I E}\left(w^{*}\right)-\kappa \ln \left(w^{*}\right)\right\} \\
& +\frac{1}{\sin \left(\theta_{\text {so }}\right)} \kappa \int d w^{*} \ln \left(w^{*}\right)
\end{aligned}
$$

The first term on the right-hand-side of (35) can be evaluated accurately using standard numerical integration rules, while the integral in the second term is computed using

$$
\frac{d}{d w^{*}}\left\{w^{*} \ln \left(w^{*}\right)-w^{*}\right\}=\ln \left(w^{*}\right) .
$$

\section{Overlapping triangles}

We first consider evaluating integrals associated with the MFIE. When $P_{o}$ and $P_{S}$ coincide with each other, the MFIE integral $I^{M F I E}$ in (10) simplifies to

$$
I^{M F I E}=\left\{\begin{array}{cc}
-\frac{1}{2} \iint_{P_{o}} d s\left(\mathbf{r}_{o}-\mathbf{r}_{o b}\right) \cdot\left(\mathbf{r}_{o}-\mathbf{r}_{s b}\right) & \text { when } l=0 \\
0 & \text { when } l \neq 0
\end{array} .\right.
$$

This integral can be evaluated analytically, as


$$
\begin{aligned}
\iint_{P_{o}} d s\left(\mathbf{r}_{o}-\mathbf{r}_{o b}\right) \cdot\left(\mathbf{r}_{o}-\mathbf{r}_{s b}\right) & =\iint_{P_{o}} d s\left(\mathbf{r}_{o} \cdot \mathbf{r}_{o}\right) \\
& -\left(\mathbf{r}_{o b}+\mathbf{r}_{s b}\right) \iint_{P_{o}} d s \mathbf{r}_{o}, \\
& +\left(\mathbf{r}_{o b} \cdot \mathbf{r}_{s b}\right) \iint_{P_{o}} d s
\end{aligned}
$$

$$
\begin{aligned}
\iint_{P_{o}} d s\left(\mathbf{r}_{o} \cdot \mathbf{r}_{o}\right)=\frac{A_{o}}{6} & \left\{\left(\mathbf{r}_{o 1} \cdot \mathbf{r}_{o 1}\right)+\left(\mathbf{r}_{o 2} \cdot \mathbf{r}_{o 2}\right)+\left(\mathbf{r}_{o 3} \cdot \mathbf{r}_{o 3}\right)\right. \\
& \left.+\left(\mathbf{r}_{o 1} \cdot \mathbf{r}_{o 2}\right)+\left(\mathbf{r}_{o 1} \cdot \mathbf{r}_{o 3}\right)+\left(\mathbf{r}_{o 2} \cdot \mathbf{r}_{o 3}\right)\right\}
\end{aligned}
$$

$\iint_{P_{O}} d s \mathbf{r}_{o}=\frac{A_{O}}{3}\left(\mathbf{r}_{o 1}+\mathbf{r}_{o 2}+\mathbf{r}_{o 3}\right)$,

and

$$
\iint_{P_{o}} d s=A
$$

Here, $\mathbf{r}_{o 1}, \mathbf{r}_{o 2}$, and $\mathbf{r}_{o 3}$ are the position vectors of $P_{o}$ 's three vertices, and $A_{O}$ is its surface area.

Next, we present an exact scheme for evaluating the EFIE integrals in the "overlapping triangles" scenario. The scheme improves on the approximate method in [15].

To analytically evaluate the two EFIE integrals in (8) and (9), a new $(u, v, w)$ coordinate system shown in Fig. 4 is adopted. In this new system, the observation and source points $\mathbf{r}_{o}$ and $\mathbf{r}_{S}$ are located at $\left(u_{o}, v_{o}, 0\right)$ and $\left(u_{s}, v_{s}, 0\right)$, respectively. In turn, the integrals in (8) and (9) are expressed as

$$
\begin{array}{r}
I_{1}^{E F I E}=\int_{-\infty}^{+\infty} \int_{-\infty}^{+\infty} d u_{o} d v_{o} \Pi^{2 \mathrm{D}}\left(u_{o}, v_{o}\right)\left(\mathbf{r}_{o}-\mathbf{r}_{o b}\right) \\
\int_{-\infty}^{+\infty} \int_{-\infty}^{+\infty} d u_{s} d v_{s} \Pi^{2 \mathrm{D}}\left(u_{s}, v_{s}\right)
\end{array}
$$




$$
\begin{array}{r}
I_{2}^{E F I E}=\int_{-\infty}^{+\infty} \int_{-\infty}^{+\infty} d u_{o} d v_{o} \Pi^{2 \mathrm{D}}\left(u_{o}, v_{o}\right) \\
\int_{-\infty}^{+\infty} \int_{-\infty}^{+\infty} d u_{s} d v_{s} \Pi^{2 \mathrm{D}}\left(u_{s}, v_{s}\right) \\
\frac{\int_{-\infty}^{t_{l}-R / c} T\left(t^{\prime}\right) d t^{\prime}}{R}
\end{array}
$$

Using variable transformations $u=u_{o}-u_{s}$ and $v=v_{o}-v_{s}$, (42) and (43) are transformed into

$$
\begin{aligned}
& I_{1}^{E F I E}=\int_{-\infty}^{+\infty} \int_{-\infty}^{+\infty} d u d v \frac{\left[\frac{d}{d t^{\prime}} T\left(t^{\prime}\right)\right]_{t^{\prime}=t_{l}-r / c}}{r} \Omega_{1}^{\text {self }}(u, v), \\
& I_{2}^{E F I E}=\int_{-\infty}^{+\infty} \int_{-\infty}^{+\infty} d u d v \frac{\int_{-\infty}^{t_{l}-r / c} T\left(t^{\prime}\right) d t^{\prime}}{r} \Omega_{2}^{\text {self }}(u, v),
\end{aligned}
$$

where

$$
\begin{aligned}
& \Omega_{1}^{\text {self }}(u, v)=\int_{-\infty}^{+\infty} \int_{-\infty}^{+\infty} d u_{o} d v_{o} \\
& \Pi^{2 \mathrm{D}}\left(u_{o}, v_{o}\right) \Pi^{2 \mathrm{D}}\left(u_{o}-u, v_{o}-v\right), \\
& \left(\mathbf{r}_{o}-\mathbf{r}_{o b}\right) \cdot\left(\mathbf{r}_{o}-\mathbf{r}-\mathbf{r}_{s b}\right) \\
& \Omega_{2}^{\text {self }}(u, v)=\int_{-\infty}^{+\infty} \int_{-\infty}^{+\infty} d u_{o} d v_{o} \\
& \Pi^{2 \mathrm{D}}\left(u_{o}, v_{o}\right) \Pi^{2 \mathrm{D}}\left(u_{o}-u, v_{o}-v\right)
\end{aligned}
$$

$\mathbf{r}=u \hat{\mathbf{u}}+v \hat{\mathbf{v}}$, and $r=\sqrt{u^{2}+v^{2}}$.

The integral in (44) can be interpreted as the field at $(0,0,0)$ due to a source spanning domain $\Omega_{1}^{\text {self }}$; similarly, the integral in (45) can be viewed as the field at $(0,0,0)$ due to a source in domain $\Omega_{2}^{\text {self }}$. Functions $\Omega_{1}^{\text {self }}$ and $\Omega_{2}^{\text {self }}$ are due to spatial self-correlations (Fig. 4). They are slightly more intricate than $\Omega_{1}^{E F I E}(u, v, w)$ and $\Omega_{2}^{E F I E}(u, v, w)$ in (26) and (27), but share their main properties. Specifically, $\Omega_{1}^{\text {self }}$ and $\Omega_{2}^{\text {self }}$ are zero outside a planar polygon region that consists of six triangles (Fig. 4). Over each of the six triangles, $\Omega_{1}^{\text {self }}$ and $\Omega_{2}^{\text {self }}$ can be expressed as multinomials involving terms of the form $u^{i} v^{j}$, where $i$ and $j$ are integers with values $0,1,2, \ldots$. Also, $\left[d T\left(t^{\prime}\right) / d t^{\prime}\right]_{t^{\prime}=t_{l}-r / c}$ and $\int_{-\infty}^{t_{l}-r / c} T\left(t^{\prime}\right) d t^{\prime}$ can be expressed as linear combinations of $r^{\tau}$, where $\tau$ is an integer that takes on values $0,1,2, \ldots$. Therefore, $I_{1}^{E F I E}$ and $I_{2}^{E F I E}$ in (44) and (45) can be decomposed into linear combinations of integrals of the form $\iint\left(r^{\tau} u^{i} v^{j}\right) d u d v \quad, \quad$ with $\tau=-1,0,1,2, \ldots \quad$ and $i, j=0,1,2, \ldots$, which can be evaluated analytically following the procedure in [15].

\section{NUMERICAL RESUltS AND ANALYSIS OF SPURIOUS MODES}

This section presents numerical results to demonstrate the accuracy and stability of the proposed EFIE, MFIE, and CFIE (with $\xi=0.5$ ) MOT solvers. In all examples presented below, the incident plane wave is parametrized as

$$
\mathbf{E}^{i}(\mathbf{r}, t)=\hat{\mathbf{p}}^{i} e^{\frac{-\left(t-t_{p}-\mathbf{r} \cdot \hat{\mathbf{k}}^{i} / c\right)^{2}}{2 \sigma^{2}}} \cos \left[2 \pi f_{0}\left(t-\mathbf{r} \cdot \hat{\mathbf{k}}^{i} / c\right)\right],
$$

where $\hat{\mathbf{k}}^{i}$ and $\hat{\mathbf{p}}^{i}$ denote the wave's propagation direction and polarization vector, $t_{p}=10 \sigma$ ensures that the system is quasi-quiescent before $t=0$, and $f_{0}$ is the incident wave's center frequency. With these choices, more than $99.99 \%$ of the incident wave's energy is contained in the frequency band $\left[f_{0}-3 /(2 \pi \sigma), f_{0}+3 /(2 \pi \sigma)\right]$; in what follows, we denote the bandwidth of the excitation as $6 /(2 \pi \sigma)$.

The EFIE, MFIE, and CFIE MOT solvers are applied to the analysis of scattering from three canonical geometries: a sphere, a circular cylinder, and a rectangular box. Because the cavity modes associated with these three geometries are known analytically, they facilitate rigorous investigations of the spurious modes affecting MOT solutions.

\section{A. Sphere scatterer}

Consider a PEC sphere with radius 1 meter and with its center at the spatial origin, which is illuminated by a plane wave with $\hat{\mathbf{k}}^{i}=\hat{\mathbf{z}}, \hat{\mathbf{p}}^{i}=\hat{\mathbf{x}}, f_{0}=114 \mathrm{MHz}$, and bandwidth of $227 \mathrm{MHz}$. The surface of the sphere is discretized using $N_{s}=$ 1,287 spatial basis functions and $\Delta t=0.44 \mathrm{~ns}$.

Fig. 5 shows the $\theta$-component of $\mathbf{J}$ at $x=1 \mathrm{~m}, y=0$, and $z=0$ obtained using the Mie series as well as the EFIE, MFIE, and CFIE MOT solvers. The solutions obtained using the three integral equation solvers agree with the Mie series solutions.

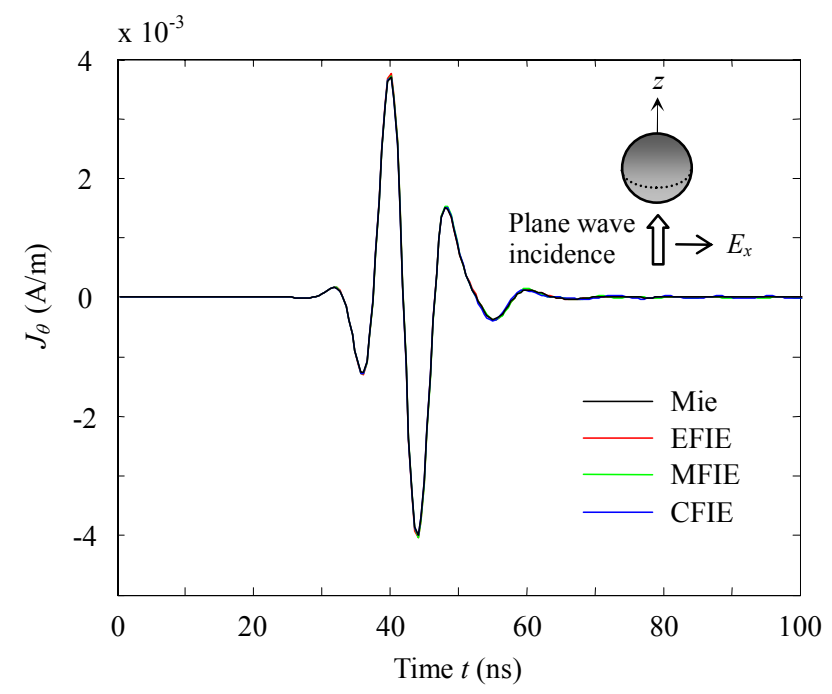

Fig. 5. Mie series solution and MOT solutions of $J_{\theta}$, at an observation point over a sphere scatterer.

The errors in the MOT solutions are analyzed in more detail in Fig. 6. The difference between the MOT solutions and Mie series solution in Fig. 5 is displayed in Fig. 6(a). The MOT matrix elements were evaluated to at least 3 digits of accuracy and the error of MOT solutions is dominated by the discretization in space and time. The representation of the current density in space and time is directly related to the accuracy of the MOT scheme. The oscillatory nature of the current density relates to its temporal bandwidth, and dictates the rate of spatial variations as well. It follows that to decrease the error, current expansions need to be simultaneously refined in both space and time. As a result, commensurate discretization in space and time are required to avoid wasting 
computational resources. Given a rate of discretization in space and time, the errors of EFIE, MFIE, and CFIE MOT solutions are observed to be on the same order in Fig. 6(a). These errors can be reduced by adjusting the spatial and temporal discretization rates. Figures 6(b) and 6(c) plot errors for $\left(N_{s}=\right.$ 2,202 RWG basis functions \& $\Delta t=0.34 \mathrm{~ns})$ and $\left(N_{s}=4,140\right.$ RWG basis functions \& $\Delta t=0.25 \mathrm{~ns}$ ). As expected, increasing the number of RWG basis functions and decreasing the time step size $\Delta t$ reduce the errors of the MOT solutions. The MOT solvers in this paper are not "fast," as their CPU usage scales proportional to $\left(N_{s}\right)^{2} N_{t}$. In Fig. 6(a), $N_{s}=1,287$ and $\Delta t=0.44 \mathrm{~ns}$; and in Fig. $6(\mathrm{c}), N_{s}=4,410$ and $\Delta t=0.25 \mathrm{~ns}$. As a result, with reference to Fig. 6(a), the CPU time consumption of Fig. 6(c) increases by a factor of

$$
\left(\frac{4410}{1287}\right)^{2} \times\left(\frac{0.44}{0.25}\right) \approx 20 \text {. }
$$
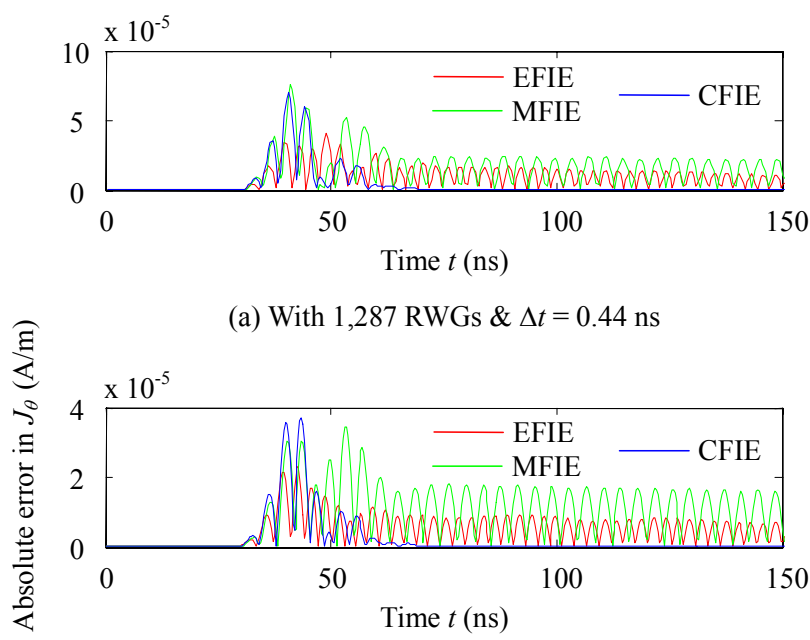

(b) With 2,202 RWGs \& $\Delta t=0.34 \mathrm{~ns}$

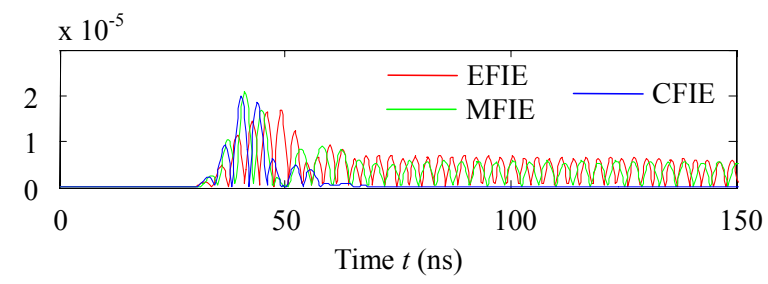

(c) With 4,140 RWGs \& $\Delta t=0.25 \mathrm{~ns}$

Fig. 6. Error of MOT solutions in $J_{\theta}$, at an observation point over a sphere scatterer.

The L2 norm error associated with the solution in Fig. 6(a), defined as

$$
\text { L2 norm error }=\sqrt{\frac{\int_{0}^{100 \mathrm{~ns}}\left\{J_{\theta}^{\mathrm{Mie}}(t)-J_{\theta}^{\mathrm{CFIE}}(t)\right\}^{2} d t}{\int_{0}^{100 \mathrm{~ns}}\left\{J_{\theta}^{\mathrm{Mie}}(t)\right\}^{2} d t}},
$$

where $J_{\theta}^{\mathrm{Mie}}$ and $J_{\theta}^{\mathrm{CFIE}}$ are the Mie and CFIE MOT solutions, is calculated to be 0.017 . The error in Fig. 6(a) is obtained using
$N_{w}=7$ (with $N_{w}$ as the number of quadrature points along $w^{*}$ direction). It is noted that all integrations in our scheme are evaluated analytically, except for the one along the $w^{*}$ direction. As a result, $N_{w}$ is the only tunable parameter in our MOT solvers once the spatial mesh and temporal step size are determined. To reveal the impact of $N_{w}$ on the performance of the MOT solver, the L2 norm errors are tabulated in Table I when $N_{w}$ varies from 2 to 8 . The numerical error drops and the CPU time grows while increasing $N_{w}$. It is our experience that for standard spatial and temporal MOT discretization rates the optimal trade-off between numerical error and CPU time is achieved when $N_{w}=7$.

TABLE I: PERFORMANCE OF CFIE MOT SOLVER WITH RESPECT TO THE NUMBER OF QUADRATURE POINTS

\begin{tabular}{ccc}
\hline \hline $\begin{array}{c}N_{w} \text {, number of } \\
\text { quadrature points } \\
\text { along } w^{*}\end{array}$ & $\begin{array}{c}\text { L2 norm error of } \\
\text { CFIE MOT solution }\end{array}$ & $\begin{array}{c}\text { CPU time } \\
\text { consumption of } \\
\text { CFIE MOT solver }\end{array}$ \\
\hline 2 & 0.085 & 134.1 seconds \\
3 & 0.047 & 180.7 seconds \\
4 & 0.031 & 231.8 seconds \\
5 & 0.026 & 292.3 seconds \\
6 & 0.021 & 354.3 seconds \\
7 & 0.017 & 421.6 seconds \\
8 & 0.015 & 487.8 seconds \\
\hline \hline
\end{tabular}

The late-time stability of the proposed MOT solvers is demonstrated in Fig. 7, which shows stable solutions for $N_{s}=$ 1,287 and $\Delta t=0.44 \mathrm{~ns}$ well after the incident wave has ceased interacting with the sphere. The analytical Mie series solution reveals that $J_{\theta}$ at $x=1 \mathrm{~m}, y=0$, and $z=0$ becomes vanishingly small for $t>100 \mathrm{~ns}$. The CFIE MOT solution exhibits the same behavior. In contrast, the EFIE and MFIE MOT solutions decay slowly after $t=100 \mathrm{~ns}$ as they are corrupted by spurious modes. The presence of spurious modes, already quite clear in Fig. 6, is analyzed next.

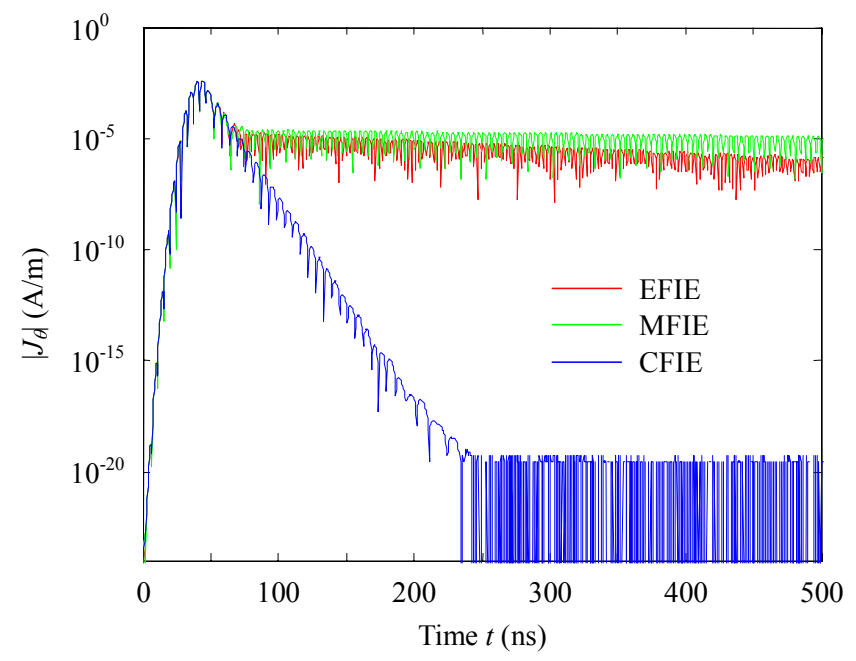

Fig. 7. Late-time data of $J_{\theta}$ at an observation point over a sphere scatterer. 
It is conjectured in [4] that MOT solutions to the time domain EFIE and MFIE may be corrupted by spurious cavity modes, but that to the CFIE is free from such erroneous contributions. To investigate this hypothesis, the late-time portions $(t>100 \mathrm{~ns})$ of the above EFIE, MFIE, and CFIE MOT solutions are Fourier transformed, and the magnitude of the resulting spectra are plotted in Fig. 8. The spikes in the resulting spectra of the EFIE and MFIE MOT solutions correspond to modes of a spherical cavity with PEC and Perfect Magnetically Conducting (PMC) walls, respectively. Let $\mathrm{TE}_{m n p}^{r}$ and $\mathrm{TM}_{m n p}^{r}$ denote transverse electric and magnetic to $r$ modes in a spherical cavity [21]. Figure 8 shows that the EFIE and MFIE solutions are possibly corrupted by currents of the cavity's (degenerate) $\mathrm{TM}_{011}^{r}, \mathrm{TM}_{111}^{r}, \mathrm{TE}_{011}^{r}$, and $\mathrm{TE}_{111}^{r}$ modes (with resonant frequency at $131 \mathrm{MHz}), \mathrm{TM}_{021}^{r}, \mathrm{TM}_{121}^{r}, \mathrm{TM}_{221}^{r}$, $\mathrm{TE}_{021}^{r}, \mathrm{TE}_{121}^{r}$, and $\mathrm{TE}_{221}^{r}$ modes (at $185 \mathrm{MHz}$ ), and $\mathrm{TM}_{011}^{r}$, $\mathrm{TM}_{111}^{r}, \mathrm{TE}_{011}^{r}$, and $\mathrm{TE}_{111}^{r}$ modes (at $215 \mathrm{MHz}$ ). Meanwhile, the CFIE MOT solutions are free of spurious cavity modes. These observations corroborate the hypothesis in [4].

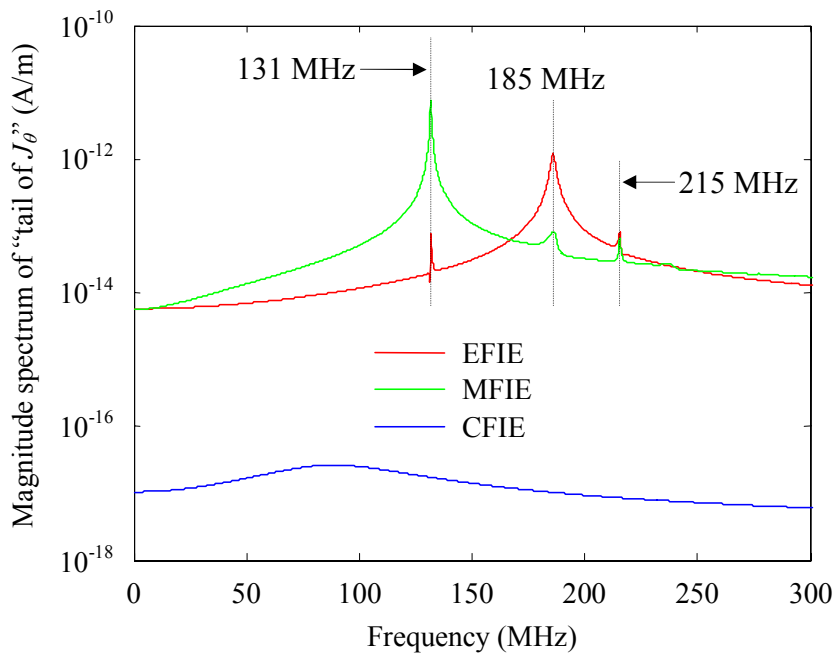

Fig. 8. Magnitude spectra of "temporal tails of $J_{\theta}$," at an observation point over a sphere scatterer.

\section{B. Circular cylinder scatterer}

Next, consider a PEC circular cylinder with radius $0.3 \mathrm{~m}$ and height $1.7 \mathrm{~m}$, which is illuminated by a plane wave with $\hat{\mathbf{k}}^{i}=\hat{\mathbf{x}}$, $\hat{\mathbf{p}}^{i}=\hat{\mathbf{z}}, f_{0}=241 \mathrm{MHz}$, and bandwidth $481 \mathrm{MHz}$. The cylinder's surface is discretized using $N_{s}=1,740 \mathrm{RWG}$ basis functions and $\Delta t=0.1 \mathrm{~ns}$.

Figure 9 shows the $x$-component of $\mathbf{J}$ at $x=0.101 \mathrm{~m}, y=$ $0.0433 \mathrm{~m}$, and $z=1.7 \mathrm{~m}$ obtained using the EFIE, MFIE, and CFIE MOT solvers. The three solutions match each other very well. Late-time stability of the three solutions is clearly demonstrated in Fig. 10.

The temporal tails of the above currents $(t>80 \mathrm{~ns})$ were Fourier transformed, and the resulting spectra are plotted in Fig. 11. There is a spike in the spectrum of the EFIE current at 306 $\mathrm{MHz}$, which is associated with the $\mathrm{TE}_{111}^{z}$ mode in a cavity with PEC wall. Likewise, the spike in the MFIE current at 306 $\mathrm{MHz}$ is due to the $\mathrm{TM}_{111}^{z}$ mode in a cavity with PMC wall. Here $\mathrm{TE}_{m n p}^{z}$ and $\mathrm{TM}_{m n p}^{z}$ denote transverse electric and magnetic to $z$ modes in a circular cylindrical cavity as defined in [21]. In this example, the incident electric field is $z$-polarized. If the cylinder scatterer were infinitely long along $z$, the scattered fields would only include $\mathrm{TM}^{z}$ modes (in other words, $\mathrm{TE}^{z}$ modes would not be excited). As the scatterer is fairly long along $z$ relative to its $x-y$ dimensions, $\mathrm{TM}^{z}$ modes are excited more strongly than their $\mathrm{TE}^{z}$ counterparts. Specifically, the spurious spike associated with the $\mathrm{TM}_{111}^{z}$ mode is two orders of magnitude stronger than that associated with the $\mathrm{TE}_{111}^{z}$ mode (Fig. 11). Also because of the above argument, the spurious mode corresponding to the $\mathrm{TM}_{010}^{z}$ mode (with PEC wall) in the late-time EFIE MOT solution at $382 \mathrm{MHz}$ is far more strongly excited than the (virtually undetectable) $\mathrm{TE}_{010}^{z}$ cavity mode (with PMC wall) in the late-time MFIE MOT solution. The frequencies of the other spurious spikes in Fig. 11 all match the theoretical cavity modes' resonant frequencies very well. For instance, the spurious spikes located at $342 \mathrm{MHz}$ in Fig. 11 correspond to $\mathrm{TM}_{112}^{z}$ and $\mathrm{TE}_{112}^{z}$ modes, respectively. Likewise, the spurious mode at $395 \mathrm{MHz}$ in the MFIE current's spectrum is due to the $\mathrm{TM}_{113}^{z}$ mode. Importantly, the solution of CFIE MOT solver does not exhibit any spurious modes.



Fig. 9. MOT solution of $J_{x}$, at an observation point over a circular cylinder scatterer.

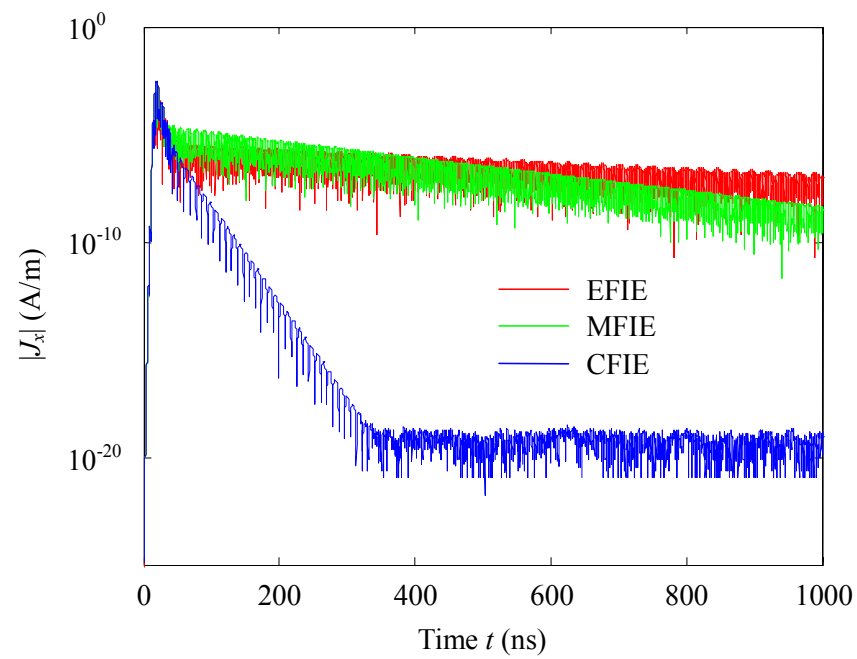

Fig. 10. Late-time data of $J_{x}$ at an observation point over a circular cylinder scatterer. 


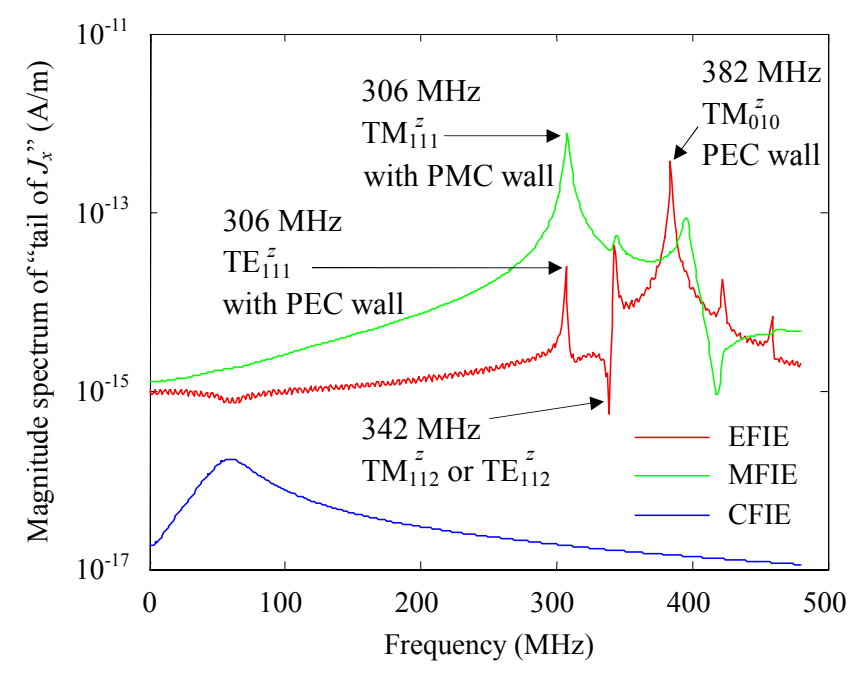

Fig. 11. Magnitude spectra of "temporal tails of $J_{x}$," at an observation point over a circular cylinder scatterer.

\section{Rectangular box scatterer}

In this subsection, consider a rectangular box measuring $1 \mathrm{~m}$ by $1 \mathrm{~m}$ by $1.7 \mathrm{~m}$ along the $x, y$, and $z$ directions, which is illuminated by a plane wave with $\hat{\mathbf{k}}^{i}=\hat{\mathbf{x}}, \hat{\mathbf{p}}^{i}=\hat{\mathbf{z}}$, $f_{0}=167 \mathrm{MHz}$, and bandwidth of $335 \mathrm{MHz}$. The box's surface is discretized using $N_{s}=1,971$ RWG basis functions and $\Delta t=0.3 \mathrm{~ns}$.

The $x$ component of $\mathbf{J}$ at $x=0.3 \mathrm{~m}, y=0.1 \mathrm{~m}$, and $z=1.7 \mathrm{~m}$ obtained using the EFIE, MFIE, and CFIE MOT solvers is shown in Fig. 12. Figure 13 depicts the magnitude of the same current component for over 3,000 time steps. Clearly, all three solvers produce similar and stable solutions.

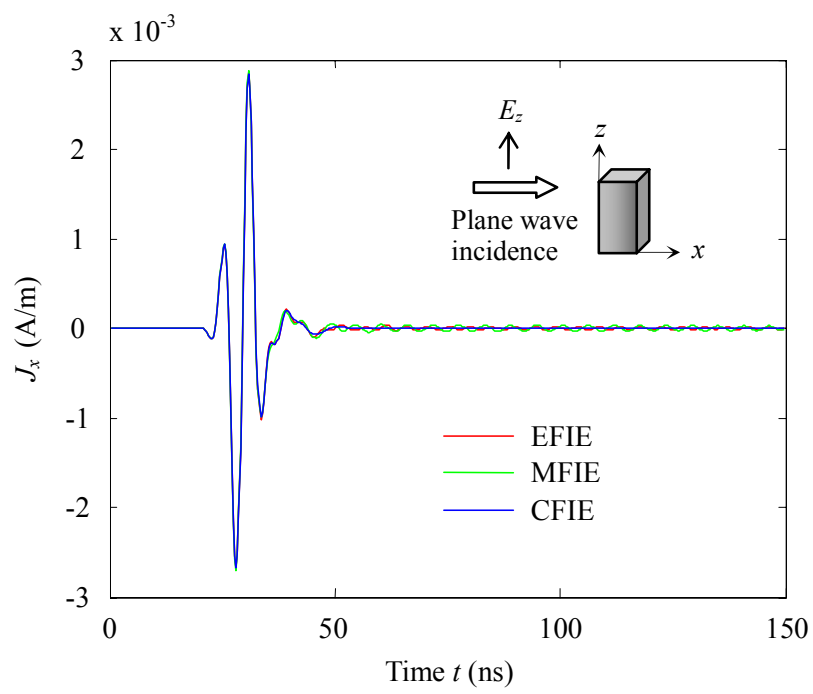

Fig. 12. MOT solution of $J_{x}$, at an observation point over a rectangular box scatterer.

The currents' temporal tails ( $t>100 \mathrm{~ns})$ once again were Fourier transformed, and the resulting spectra are shown in Fig. 14. Just like in the previous two examples, the late-time EFIE and MFIE MOT solutions are contaminated by spurious modes. Specifically, the late-time EFIE solution contains contributions from the $\mathrm{TM}_{110}^{z}$ mode in a cavity with PEC wall (at $212 \mathrm{MHz}$ ), while that of the MFIE is corrupted by currents from the $\mathrm{TM}_{011}^{z}$ and $\mathrm{TM}_{101}^{z}$ modes in a cavity with PMC wall (at 174 $\mathrm{MHz}) ; \mathrm{TM}_{m n p}^{z}$ denotes the transverse magnetic to $z$ mode in a rectangular cavity as defined in [21]. The other spikes in Fig. 14 all can be attributed to certain cavity modes. The late-time solution produced by the CFIE MOT solver is not affected by any spurious modes, once again confirming the hypothesis in [4].

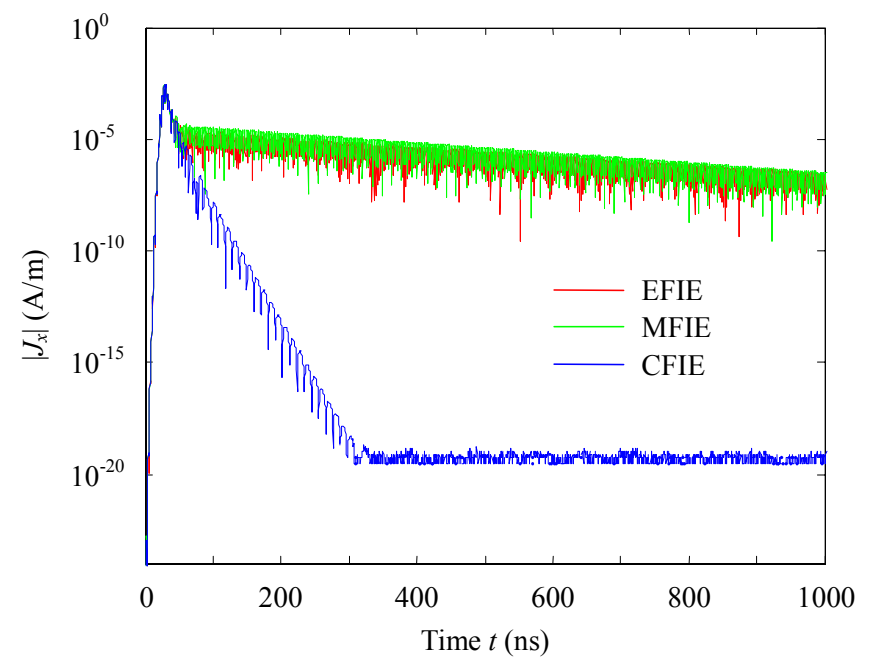

Fig. 13. Late-time data of $J_{x}$ at an observation point over a rectangular box scatterer.

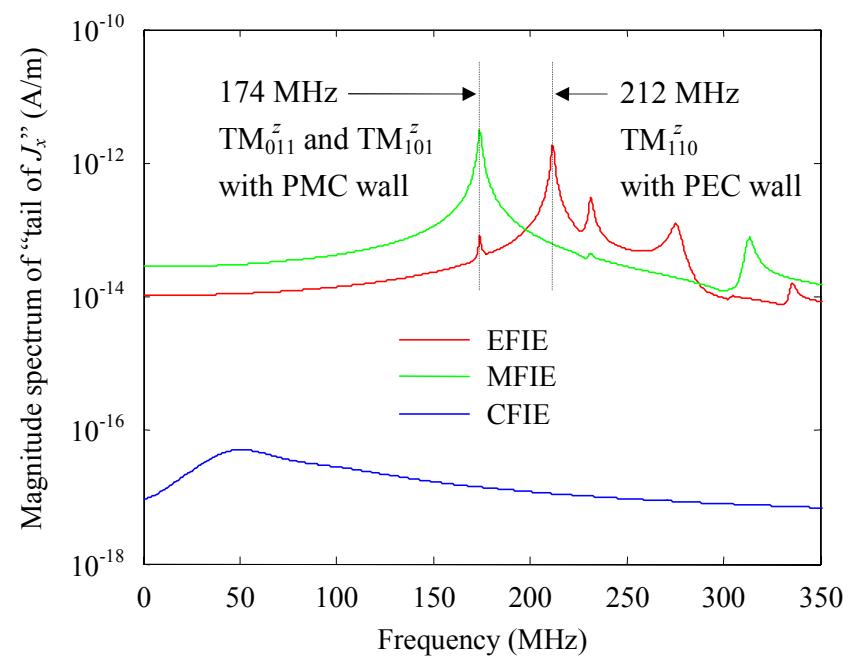

Fig. 14. Magnitude spectra of "temporal tails of $J_{x}$," at an observation point over a rectangular box scatterer.

\section{CONCLUSIONS}

This paper extends the quasi-exact integration technique in [15] to construct novel MOT solvers for the time domain EFIE, MFIE, and CFIE. The novel MOT solvers are demonstrated to be accurate and stable. Furthermore, spurious modes that potentially pollute solutions of MOT solvers are studied comprehensively. It is shown that spurious modes with frequencies matching those of cavity modes oftentimes are present in MOT solutions of both the EFIE and MFIE. In 
contrast, no spurious modes are observed in MOT solution of the CFIE. That said, we did observe peaks in CFIE solutions in Figs. 8, 11 and 14, which are several orders smaller than those associated with spurious modes. The presence of these peaks remains under investigation, and we postulate that they may be numerical artifacts. In addition, while the novel MOT scheme's stability has been validated extensively for scattering problems, its applicability to radiation and waveguide problems is being actively studied.

\section{REFERENCES}

[1] C. L. Bennett and W. L. Weeks, "Transient scattering from conducting cylinders," IEEE Transactions on Antennas and Propagation, vol. 18, no. 5, pp. 627-633, September 1970.

[2] M. J. Bluck and S. P. Walker, "Time-domain BIE analysis of large three-dimensional electromagnetic scattering problems," IEEE Transactions on Antennas and Propagation, vol. 45, no. 5, pp. 894-901, May 1997.

[3] D. A. Vechinski and S. M. Rao, "A stable procedure to calculate the transient scattering by conducting surfaces of arbitrary shape," IEEE Transactions on Antennas and Propagation, vol. 40, no. 6, pp. 661-665, June 1992.

[4] B. Shanker, A. A. Ergin, K. Aygun, and E. Michielssen, "Analysis of transient electromagnetic scattering from closed surfaces using a combined field integral equation," IEEE Transactions on Antennas and Propagation, vol. 48, no. 7, pp. 1064-1074, July 2000.

[5] R. Mittra, "Integral equation formulations and solutions of transient scattering problems," in Transient Electromagnetic Fields, L. B. Felsen, Ed. Berlin: Springer-Verlag, 1976.

[6] B. Shanker, A. A. Ergin, M. Lu, and E. Michielssen, "Fast analysis of transient electromagnetic scattering phenomena using the multilevel plane wave time domain algorithm," IEEE Transactions on Antennas and Propagation, vol. 51, no. 3, pp. 628-641, 2003.

[7] P. J. Davies, "On the stability of time-marching schemes for the general surface electric-field integral equation," IEEE Transactions on Antennas and Propagation, vol. 44, no. 11, pp. 1467-1473, November 1996.

[8] Y. Shi, H. Bagci, and M. Lu, "On the internal resonant modes in marching-on-in-time solution of the time domain electric field integral equation," IEEE Transactions on Antennas and Propagation, vol. 61, no. 8, pp. 4389-4392, August 2013.

[9] T. Abboud, J.-C. Nedelec, and J. Volakis, "Stable solution of the retarded potential equations," presented at 17th Annual Review of Progress in Applied Computational Electromagnetics, Monterey, CA, March 2001.

[10] T. Ha-Duong, "On retarded potential boundary integral equations and their discretisation," in Topics in Computational Wave Propagation: Direct and Inverse Problems, M. Ainsworth, P. Davies, D. Duncan, P. Martin, and B. Rynne, Eds. Berlin: Springer-Verlag, 2003.

[11] J. Pingenot, S. Chakraborty, and V. Jandhyala, "Polar integration for exact space-time quadrature in time-domain integral equations," IEEE Transactions on Antennas and Propagation, vol. 54, no. 10, pp. 3037-3042, October 2006

[12] A. C. Yucel and A. A. Ergin, "Exact evaluation of retarded-time potential integrals for the RWG bases," IEEE Transactions on Antennas and Propagation, vol. 54, no. 5, pp. 1496-1502, May 2006.

[13] B. Shanker, M. Lu, J. Yuan, and E. Michielssen, "Time domain integral equation analysis of scattering from composite bodies via exact evaluation of radiation fields," IEEE Transactions on Antennas and Propagation, vol. 57, no. 5, pp. 1506-1520, May 2009.

[14] H. A. Ulku and A. Ergin, "Application of analytical retarded-time potential expressions to the solution of time domain integral equations," IEEE Transactions on Antennas and Propagation, vol. 59, no. 11, pp. 4123-4131, November 2011.

[15] Y. Shi, M.-Y. Xia, R.-S. Chen, E. Michielssen, and M. Lu "Stable electric field TDIE solvers via quasi-exact evaluation of MOT matrix elements," IEEE Transactions on Antennas and Propagation, vol. 59, no. 2, pp. 574-585, February 2011.

[16] S. M. Rao, D. R. Wilton, and A. W. Glisson, "Electromagnetic scattering by surfaces of arbitrary shape," IEEE Transactions on Antennas and Propagation, vol. 30, no. 3, pp. 409-418, May 1982.

[17] G. Manara, A. Monorchio, and R. Reggiannini, "A space-time discretization criterion for a stable time-marching solution of the electric field integral equation," IEEE Transactions on Antennas and Propagation, vol. 45, no. 3, pp. 527-532, March 1997.

[18] L. Gurel and O. Ergul, "Singularity of the magnetic-field integral equation and its extraction," IEEE Antennas and Wireless Propagation Letters, vol. 4, pp. 229-232, 2005.

[19] B. Shanker, A. A. Ergin, K. Aygun, and E. Michielssen, "Analysis of transient electromagnetic scattering from closed surfaces using a combined field integral equation," Center for Computational Electromagnetics, University of Illinois, Urbana-Champaign, Research Report CCEM 30-98, October 6, 1998.

[20] A. A. Ergin, B. Shanker, and E. Michielssen, "Analysis of transient scattering from rigid bodies using the Burton-Miller approach," Journal of Acoustical Society of America, vol. 106, pp. 2396-2404, 1999.

[21] C. A. Balanis, Advanced Engineering Electromagnetics. Hoboken, NJ: Wiley, 1989.

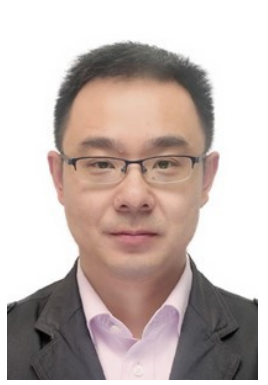

Xin Wang (S'07-M'10) received the B.S. and M.S. degrees in electronic engineering from Tsinghua University, Beijing, China in 2000 and 2002 respectively, and the $\mathrm{Ph} . \mathrm{D}$. degree in electrical and computer engineering from Purdue University, West Lafayette, IN in 2009.

He was a Post-Doctoral Research Associate with the Birck Nanotechnology Center, Purdue University from 2009 to 2010. He is currently an Associate Professor with the College of Electronic and Information Engineering, Nanjing University of Aeronautics and Astronautics, Nanjing, China. His research interest includes wireless power transmission, reconfigurable antennas, and RF circuits.

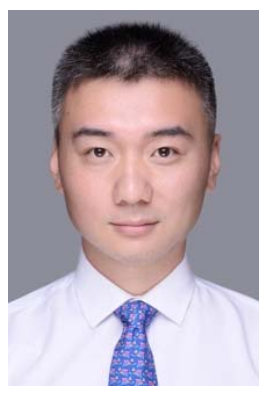

Yifei Shi received the B.Tech. degree in electronic and information engineering from Nanjing Tech University, Nanjing, China in 2004, and Ph.D. degree in electromagnetic filed and microwave technology from Nanjing University of Science and Technology, Nanjing, China in 2012.

From 2012 to 2015, he was a Postdoc Research Fellow with the Computational Electromagnetics Laboratory at King Abdullah University of Science and Technology, Thuwal, Saudi Arabia. He joined the Department of Electronic Engineering, Jiangsu University of Technology, Changzhou, Jiangsu, China as a Lecturer in 2016, and was promoted to Associate Professor in 2017. His research interest includes time domain integral equation, hybrid methods, and fast algorithms.

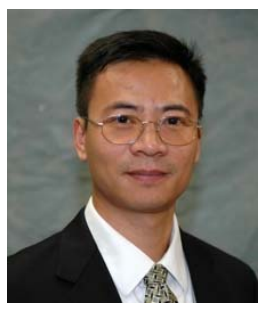

Mingyu Lu (M'03-SM'08) received the B.S. and M.S. degrees in electrical engineering from Tsinghua University, Beijing, China in 1995 and 1997 respectively, and the Ph.D. degree in electrical engineering from the University of Illinois at Urbana-Champaign in 2002.

From 2002 to 2005, he was a Postdoctoral Research Associate at the Electromagnetics Laboratory in the University of Illinois at Urbana-Champaign. $\mathrm{He}$ was an Assistant Professor with the Department of Electrical Engineering, University of Texas at Arlington from 
2005 to 2012. He joined the Department of Electrical and Computer Engineering, West Virginia University Institute of Technology in 2012 and he is currently a Professor. His research interest includes wireless power transmission, radar systems, antenna design, and computational electromagnetics.

Dr. Lu was the recipient of the first prize award in the student paper competition of IEEE Antennas and Propagation International Symposium, Boston, MA in 2001. He served as the chair of Antennas and Propagation Society of IEEE Fort Worth Chapter from 2006 to 2011, and he received the Outstanding Service Award from IEEE Fort Worth Chapter in 2008.

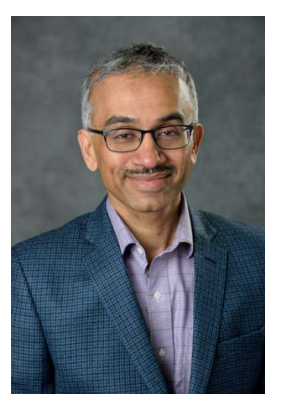

Balasubramaniam Shanker (SM'02-F'10) received his B'Tech from the Indian Institute of Technology, Madras, India in 1989, M.S. and Ph.D in 1992 and 1993, respectively, from the Pennsylvania State University.

From 1993 to 1996 he was a research associate in the Department of Biochemistry and Biophysics at Iowa State University where he worked on the Molecular Theory of Optical Activity. From 1996 to 1999 he was with the Center for Computational Electromagnetics at the University of Illinois at Urbana-Champaign as a Visiting Assistant Professor, and from 1999-2002 with the Department of Electrical and Computer Engineering at Iowa State University as an Assistant Professor. Currently, he is a University Distinguished Professor in the Department of Electrical and Computer Engineering at Michigan State University, and the Department of Physics and Astronomy. From 2015-2018, he was appointed Associate Chair of the Department of Computational Mathematics, Science and Engineering, a new department at MSU and was a key player in building this Department. Earlier he served as the Associate Chair for Graduate Studies in the Department of Electrical and Computer Engineering from 2012-2015, and currently is the Associate Chair for Research in ECE. He has authored/co-authored around 400 journal and conference papers and presented a number of invited talks. His research interests include all aspects of computational electromagnetics (frequency and time domain integral equation based methods, multi-scale fast multipole methods, fast transient methods, higher order finite element and integral equation methods), propagation in complex media, mesoscale electromagnetics, and particle and molecular dynamics as applied to multiphysics and multiscale problems.

He was an Associate Editor for IEEE Antennas and Wireless Propagation Letters (AWPL) and IEEE Transactions on Antennas and Propagation, is the Topical Editor for Journal of Optical Society of America: A, and is a full member of the USNC-URSI Commission B. He is Fellow of IEEE, elected for his contributions in computational electromagnetics. He has also been awarded the Withrow Distinguished Junior scholar (in 2003), Withrow Distinguished Senior scholar (in 2010), the Withrow teaching award (in 2007), and the Beal Outstanding Faculty award (2014).

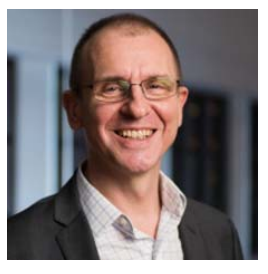

Eric Michielssen (SM'99-F'02) received his M.S. in Electrical Engineering (Summa Cum Laude) from the Katholieke Universiteit Leuven (KUL, Belgium) in 1987, and his Ph.D. in Electrical Engineering from the University of Illinois at Urbana-Champaign (UIUC) in 1992.

From 1992 to 2005, he served on the faculty at UIUC. In 2005, he joined the University of Michigan, Ann Arbor, where he currently is the Louise Ganiard Johnson Professor of Engineering and Professor of Electrical Engineering and Computer Science. Eric Michielssen authored or co-authored over 200 journal papers and book chapters and over 400 papers in conference proceedings. His research interests include all aspects of theoretical and applied computational electromagnetics. His research focuses on the development of fast frequency and time domain integral-equation-based techniques for analyzing electromagnetic phenomena, and the development of robust optimizers for the synthesis of electromagnetic/optical devices.

Eric Michielssen received a Belgian American Educational Foundation Fellowship in 1988. He was the recipient of a 1995 National Science Foundation CAREER Award and the 1998 Applied Computational Electromagnetics Society (ACES) Valued Service Award. In 1999, he was named 1999 URSI United States National Committee Henry G. Booker Fellow and selected as the recipient of the URSI Koga Gold Medal. In 2003 he served as a Scholar in the Tel Aviv University Sackler Center for Advanced Studies. He was the recipient of the 2014 IEEE AP-S Chen-To-Tai Distinguished Educator Award, the 2017 IEEE APS Sergei A. Schelkunoff Transactions Prize Paper Award, the 2020 Harrington-Mittra Computational Electromagnetics Award, and the 2020 ACES Computational Electromagnetics Award. He is a Fellow of the IEEE (elected 2002) and a member of URSI Commission B.

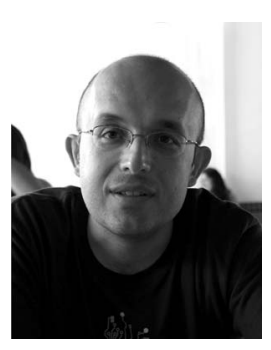

Hakan Bagci (S'98-M'07-SM'14) received the B.S. degree in Electrical and Electronics Engineering from the Bilkent University, Ankara, Turkey, in 2001; and the M.S. and Ph.D. degrees in Electrical and Computer Engineering from the University of Illinois at UrbanaChampaign (UIUC), Urbana, IL, USA, in 2003 and 2007, respectively.

From June 1999 to July 2001, he worked as an Undergraduate Researcher with the Computational Electromagnetics Group, Bilkent University. From August 2001 to December 2006, he was a Research Assistant with the Center for Computational Electromagnetics and Electromagnetics Laboratory, UIUC. From January 2007 to August 2009, he was a Research Fellow with the Radiation Laboratory, University of Michigan, Ann Arbor, MI, USA. Since August 2009, he has been with the King Abdullah University of Science and Technology (KAUST), Thuwal, Saudi Arabia, where he is currently an Associate Professor of Electrical Engineering. His research interests include various aspects of theoretical and applied computational electromagnetics with emphasis on well-conditioned frequency 
and time domain integral equation formulations and their discretization, hybrid time domain integral and differential equation solvers, accurate, stable, and efficient marching schemes for time domain solvers, stochastic characterization of electromagnetic field and wave interactions on complex geometries, and solution of two and three dimensional electromagnetic inverse scattering problem using signal processing techniques. He authored or co-authored more than 90 journal papers and more than 200 contributions in conference proceedings.

Dr. Bagci was the recipient of the 2008 International Union of Radio Scientists (URSI) Young Scientist Award and the 2004-2005 Interdisciplinary Graduate Fellowship from the Computational Science and Engineering Department, UIUC. His paper titled "Fast and Rigorous Analysis of EMC/EMI Phenomena on Electrically Large and Complex Structures Loaded With Coaxial Cables" was one of the three finalists (with honorable mention) for the 2008 Richard B. Schulz Best Transactions Paper Award given by the IEEE Electromagnetic Compatibility Society. He authored (as student) or co-authored (as student and advisor) 19 finalist/honorable mention papers in the student paper competitions at the 2005, 2008, 2010, 2014, 2015, 2016, 2017, 2018, and 2020 IEEE Antennas and Propagation Society International Symposiums and 2013, 2014, 2016, 2017, 2018, and 2019 Applied Computational Electromagnetics Society Conferences. Dr. Bagci is currently an Associate Editor for the IEEE Transactions on Antennas and Propagation, IEEE Journal on Multiscale and Multiphysics Computational Techniques, and IEEE Antennas and Propagation Magazine. 